\title{
THE
}

2015

\section{Solvent Effects in the Extraction and Detection of Polycyclic Aromatic Hydrocarbons from Complex Oils in Complex Environments}

\author{
Nicole Serio \\ University of Rhode Island \\ Mindy Levine \\ University of Rhode Island, m_levine@uri.edu
}

Follow this and additional works at: https://digitalcommons.uri.edu/chm_facpubs

The University of Rhode Island Faculty have made this article openly available.

Please let us know how Open Access to this research benefits you.

This is a pre-publication author manuscript of the final, published article.

Terms of Use

This article is made available under the terms and conditions applicable towards Open Access

Policy Articles, as set forth in our Terms of Use.

Citation/Publisher Attribution

Serio, N. \& Levine, M. J Incl Phenom Macrocycl Chem (2016) 84: 61. https://doi.org/10.1007/ s10847-015-0583-y

Available at: http://dx.doi.org/10.1007/s10847-015-0583-y 
AUTHORS: Nicole Serio ${ }^{\mathrm{a}}$ and Mindy Levine ${ }^{\mathrm{a}^{*}}$

TITLE: Solvent Effects in the Extraction and Detection of Polycyclic Aromatic Hydrocarbons from Complex Oils in Complex Environments

\title{
AUTHOR ADDRESS AND AFFILIATION:
}

Department of Chemistry, University of Rhode Island, 51 Lower College Road, Kingston, RI 02881

CORRESPONDING AUTHOR INFORMATION: Telephone: 401-874-4243; Fax: 401-874-5072; Email: mlevine@chm.uri.edu; mindy.levine@gmail.com

\begin{abstract}
Anthropogenic oil spills such as the 2010 Deepwater Horizon oil spill present a number of unique challenges in environmental remediation, detection, and monitoring of a wide range of toxicants in complex environments. We have previously reported the use of cyclodextrin derivatives to accomplish the selective extraction of polycyclic aromatic hydrocarbons (PAHs) and facilitate their detection via proximity-induced energy transfer. Reported herein is the ability of these cyclodextrins to operate in a real-world scenario: extracting PAHs from oil collected from an oil spill site and from tar ball extracts into crude seawater from the Narragansett Bay in Rhode Island. The ability of this system to operate in this complex environment highlights the practical significance of cyclodextrin-based systems, and a direct comparison of the results obtained in seawater with those obtained using a variety of aqueous solvent systems provides significant insights into the factors responsible for efficient performance.
\end{abstract}

KEYWORDS: cyclodextrin; energy transfer; oil spills; polycyclic aromatic hydrocarbons; tar balls 


\section{Graphical abstract:}

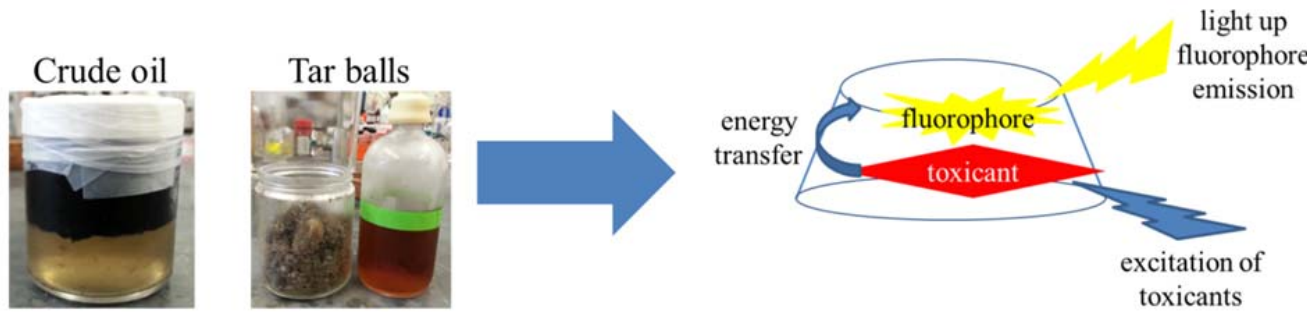




\section{INTRODUCTION}

Recent oil spills such as the 2010 Deepwater Horizon oil spill present a number of unique challenges for environmental remediation, detection, and short- and long-term monitoring of a wide range of environmental toxicants in complex environments [1]. These toxicants include $n$-alkanes, isoprenoids, and polycyclic aromatic hydrocarbons (PAHs) [2], as well as a variety of polar metabolites of these non-polar compounds, formed via facile oxidation of the original pollutants in the affected environments $[3,4]$.

Current methods used for the detection of these toxicants rely overwhelmingly on gas chromatographymass spectrometry (GC-MS) [5], which is exceptionally sensitive for non-polar toxicants but markedly insensitive to the polar oxidized metabolites. Recent research has suggested that up to $50 \%$ of the contaminants may be polar materials that are undetectable by GC-MS [6]; other methods such as liquid chromatography-mass spectrometry have been used in conjunction with GC-MS to achieve efficient detection [7].

We have previously reported a fundamentally new approach for toxicant detection and potential environmental remediation: the use of commercially available, non-toxic cyclodextrin derivatives for the efficient extraction of PAHs from oils, and for their subsequent detection via proximity-induced energy transfer [8]. This system operates successfully to extract PAHs from motor oil, vacuum pump oil, and vegetable oil, as well as from oil collected from oil spill sites and from tar balls [9]. The most efficient cyclodextrin hosts were found to be either $\gamma$-cyclodextrin, which is well-known to form ternary complexes $[10,11]$ and promote proximity-induced energy transfer [12-15] or 2-hydroxypropyl- $\beta$ cyclodextrin (2-HPCD) [16], which was substantially more efficient at extracting the PAHs compared to $\gamma$-cyclodextrin, although somewhat less efficient at promoting PAH-to-fluorophore energy transfer.

In each of our previously reported cases, the performance of cyclodextrin was tested in phosphate buffered saline (PBS) [17], which is designed to mimic the salinity of seawater while avoiding contaminants and the plethora of other compounds found in crude seawater [18,19]. Solvent composition, $\mathrm{pH}$, and salinity are expected to have significant effects in the performance of cyclodextrin-based systems for PAH extraction and detection, as such systems rely on key intermolecular forces such as hydrophobic binding [20,21] and $\pi-\pi$ stacking [22] that are known to depend substantially on such system variations $[23,24]$. Reported herein is a direct investigation of solvent effects in cyclodextrin-promoted PAH extraction and energy transfer, by using crude seawater collected from the Narragansett Bay as well as phosphate buffer (without added saline), $1 \mathrm{M}$ guanidinium hydrochloride, $1 \mathrm{M}$ sodium chloride, ultrapure water, and a water/ethanol (1:1 vol:vol) mixture. The results highlight the ability of cyclodextrin to operate successfully in seawater, and also provide novel insights into the mechanisms responsible for the observed supramolecular phenomena of PAH extraction and proximity-induced energy transfer.

\section{EXPERIMENTAL}

For full details of all experimental procedures, please see the Electronic Supporting Information.

\section{Materials and Methods}

Two oil samples were analyzed: Oil collected from an oil spill site (collected in Louisiana, April 2012), and tar ball oil (collected in Alabama, November 2013). Compounds 1-3 were purchased from Sigma Aldrich Company and used as received (Figure 1). Compound 4 was synthesized following literaturereported procedures [25]. Spectra/Por ${ }^{\circledR} 2$ Dialysis membranes (Flat Width $45 \mathrm{~mm}$, MWCO 12-14 kD) were purchased from Fisher Scientific and rinsed in deionized water for 15 to 20 minutes, in accordance with the product instructions. Fluorescence measurements were recorded on a Shimadzu RF5301 spectrophotofluorimeter, with a $1.5 \mathrm{~nm}$ excitation slit width and a $1.5 \mathrm{~nm}$ emission slit width. All spectra were integrated versus wavenumber on the X-axis. Seawater was collected from the Narragansett Bay in Rhode Island in May 2014, at a depth of 2 meters. 


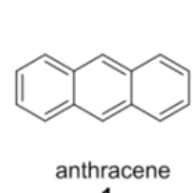

1

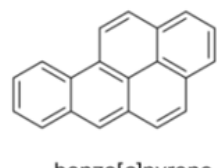

2

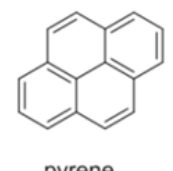

3

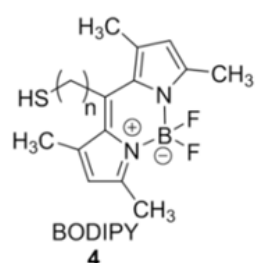

4

Fig 1 Structures of analytes (1-3) and fluorophore 4

\section{Oil Extraction Experimental Procedures}

To prepare the oil spill oil, the oil was diluted in a $1: 4$ ratio with $n$-hexanes $(0.625 \mathrm{~mL}$ of oil spill oil and $1.875 \mathrm{~mL}$ of $n$-hexanes). The tar balls were prepared by placing a tar ball in a mortar and pestle and breaking it up mechanically. Then, $\sim 5 \mathrm{~mL}$ of hexanes was added and the tar balls were mixed into the hexanes solution. Next, the solution was placed in a dialysis bag and placed in a beaker with $\sim 400 \mathrm{~mL} n$ octane. The sample was allowed to dialyze for 3 days until the octane turned brown in color. After this time, the bag was removed and the resulting octane solution was centrifuged at $3000 \mathrm{rpm}$ for 10 minutes. The brown solution was then decanted and stored as the tar ball extract solution. For each experiment, 2.5 $\mathrm{mL}$ of this stock solution was used.

PAH extraction experiments were conducted as follows: For all experiments, $2.5 \mathrm{~mL}$ of the oil sample was mixed with $20 \mu \mathrm{L}$ of a $1 \mathrm{mg} / \mathrm{mL}$ solution of each analyte (1-3) in tetrahydrofuran (THF). The samples were vigorously shaken by hand for 1 minute, and the oil mixtures were then added to a $2.5 \mathrm{~mL}$ aqueous solution of either a $10 \mathrm{mM}$ in seawater cyclodextrin derivative ( $\alpha$-cyclodextrin, $\beta$-cyclodextrin, methyl- $\beta$-cyclodextrin, 2-hydroxypropyl- $\beta$-cyclodextrin (2-HPCD), and $\gamma$-cyclodextrin) or a $0 \mathrm{mM}$ cyclodextrin solution in seawater (control). The mixture was vigorously shaken by hand for 1 minute to ensure thorough mixing. The layers were allowed to sit undisturbed for 16-24 hours. The layers were separated and the analytes in each layer were detected by fluorescence spectroscopy with $360 \mathrm{~nm}$ excitation. The analyte fluorescence emission spectrum was integrated versus wavenumber on the $\mathrm{X}$ axis (using OriginPro 9.1 software). The amount of analyte in each layer was quantified as an "analyte comparison" and calculated according to Equation 1:

Analyte comparison $=I_{\text {aqueous }} /\left(I_{\text {aqueous }}+I_{\text {oil }}\right) \times 100 \%$

where $I_{\text {aqueous }}$ is the integrated emission of the analyte in the aqueous layer and $I_{\text {oil }}$ is the integrated emission of the analyte in the oil layer.

The ability of the cyclodextrin host to efficiently extract fluorophore 4 from the oil layer into the aqueous layer was quantified according to Equation 2,

Fluorophore ratio $=I_{\mathrm{CD}} / I_{\text {blank }}$

where $I_{\mathrm{CD}}$ is the integrated emission of fluorophore $\mathbf{4}$ in the aqueous layer with a cyclodextrin host and $I_{\text {blank }}$ is the integrated emission of fluorophore $\mathbf{4}$ in the aqueous layer without a cyclodextrin host

\section{Energy Transfer Experimental Procedures}

To a $2.5 \mathrm{~mL}$ solution of oil was added $100 \mu \mathrm{L}$ of compound $4(0.1 \mathrm{mg} / \mathrm{mL}$ in THF $), 20 \mu \mathrm{L}$ of the analyte of interest $(1.0 \mathrm{mg} / \mathrm{mL}$ in THF) and $2.5 \mathrm{~mL}$ of aqueous solution $(10 \mathrm{mM}$ or $0 \mathrm{mM}$ cyclodextrin derivative solution in seawater). The layers were vigorously shaken in a vial for 1 minute and the layers were allowed to separate for 16-24 hours. The layers were separated and each layer was excited at two wavelengths: the analyte excitation wavelength $(360 \mathrm{~nm})$ and the fluorophore excitation wavelength (460 $\mathrm{nm}$ ). Each fluorescence emission spectrum was integrated versus wavenumber on the $\mathrm{X}$ axis (using OriginPro 9.1 software). The efficiency of the energy transfer from the analytes to the fluorophore was calculated according to Equation 3: 
where $I_{\mathrm{DA}}$ is the integration of the fluorophore emission from analyte excitation and $I_{\mathrm{A}}$ is the integrated fluorophore emission from direct excitation (Figure 2).

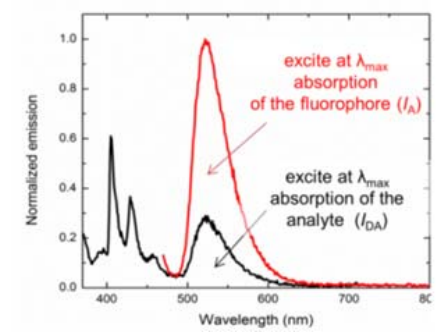

Fig 2 Illustration of energy transfer efficiency for a generic donor-acceptor combination

Additionally, energy transfer from the oil directly was measured by omitting the analyte from the above procedure. After the aqueous and oil layers were separated, the oil layer was excited at $360 \mathrm{~nm}$ (but in the absence of any doped analyte) and at the fluorophore's excitation wavelength (460 nm). Equation 3 was used to quantify the resulting energy transfer.

\section{RESULTS AND DISCUSSION}

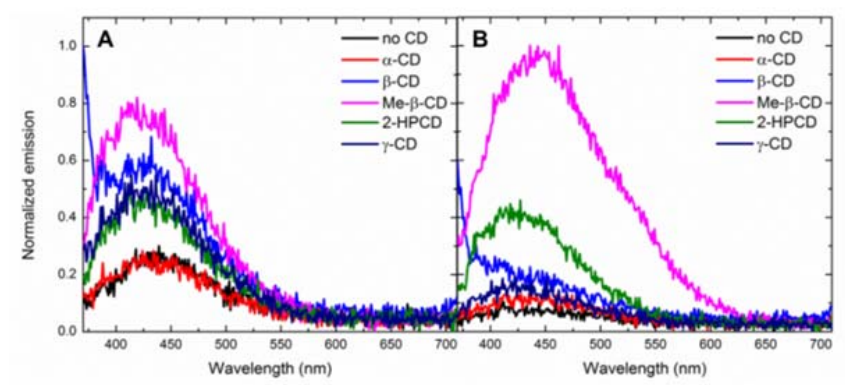

Fig 3 A comparison of the amount of fluorescent analyte extracted from the oil layer into the aqueous layer for (A) oil spill oil and (B) tar ball oil (360 nm excitation) (No analyte added to these solutions)

Our investigations focused on crude oil samples collected from oil spill sites and crude seawater from the Narragansett Bay, with $10 \mathrm{mM}$ of various cyclodextrins dissolved in the seawater. The chemical profile of oil spill oil collected directly from a Gulf of Mexico site has been well characterized in a number of references [26,27] and been shown to contain a diverse mixture of organic compounds including polycyclic aromatic hydrocarbons and a variety of more polar oxygenated metabolites [28]. Tar ball components have been similarly well-characterized using a variety of spectroscopic techniques [29-31].

As shown in Figure 3, the cyclodextrin hosts showed varying abilities to remove photophysically active components from the crude oil into the seawater, with the methyl- $\beta$-cyclodextrin solution leading to the highest amount of fluorescence signal in the aqueous layer. $\alpha$-Cyclodextrin, whose cavity size is too small to accommodate most aromatic guests, showed a fluorescence response that was indistinguishable from the cyclodextrin-free solution for both oil spill oil and tar ball oil. The cyclodextrin responses varied to some extent between the two crude oil sources, with $\beta$-cyclodextrin markedly more efficient at extracting photophysically active compounds from oil spill oil compared to the tar ball oil. This differential behavior may be related to the fact that oil spill oil is known to contain a higher fraction of oxidized PAH metabolites as a result of the weathering process. These oxidized metabolites, in turn, are best suited to bind in a $\beta$-cyclodextrin host, which has a known propensity to form strong hydrogen bonds with small molecule guests. The strong performance of methyl- $\beta$-cyclodextrin in promoting the extraction of fluorescent contaminants from crude oil into crude seawater is likely due to its strongly hydrophobic 
character which promotes binding of hydrophobic small molecule guests that are responsible for the observed fluorescence signals.

Another way to measure the efficiency of cyclodextrin hosts in extracting aromatic analytes into the aqueous layer is to quantify the ratio of fluorescence found in the aqueous layer to the total observed fluorescence using Equation 1, and the results of these calculations are summarized in Table 1. By this metric as well, methyl- $\beta$-cyclodextrin was found to be the most efficient host for analyte extraction, although $\beta$-cyclodextrin, 2-hydroxypropyl- $\beta$-cyclodextrin, and $\gamma$-cyclodextrin all led to higher amounts of photophysically active compounds in the aqueous layer compared to what was seen in the cyclodextrinfree solution, as a result of their ability to bind hydrophobic, fluorescent small molecule guests.

Table 1: Analyte comparisons in undoped solutions ${ }^{a b}$

\begin{tabular}{|c|c|c|}
\hline Host & Oil spill oil & Tar ball oil \\
\hline no cyclodextrin & $65.5 \pm 0.7$ & $48.9 \pm 0.6$ \\
\hline$\alpha$-cyclodextrin & $64.1 \pm 0.7$ & $51.5 \pm 0.2$ \\
\hline$\beta$-cyclodextrin & $78.0 \pm 0.2$ & $62.3 \pm 0.3$ \\
\hline methyl- $\beta$-cyclodextrin & $79.3 \pm 0.3$ & $81.5 \pm 0.2$ \\
\hline 2-hydroxypropyl- $\beta$-cyclodextrin & $73.1 \pm 0.9$ & $70.4 \pm 0.1$ \\
\hline$\gamma$-cyclodextrin & $74.0 \pm 0.2$ & $56.1 \pm 0.3$ \\
\hline
\end{tabular}

$a$ All analyte comparisons were calculated according to Equation 1.

$b$ The reported results represent an average of 4 trials.

Similarly, methyl- $\beta$-cyclodextrin was most efficient at extracting fluorophore 4 from the oil layer into a cyclodextrin-containing seawater solution (Figure 4) for both oil spill oil and tar ball oil samples. This fluorophore has been used previously by our group as a highly efficient fluorescent acceptor in cyclodextrin-promoted energy transfer schemes [12-16], and results using this fluorophore have been shown to be virtually indistinguishable from results obtained with a less functionalized BODIPY acceptor [see ESI for ref. 14]. 2-hydroxypropyl- $\beta$-cyclodextrin was also somewhat efficient for this extraction, with other cyclodextrin hosts showing markedly less efficient behaviors. These results are in line with our previously reported data showing that methyl- $\beta$-cyclodextrin was the most efficient host for extraction in purified buffer solution [9], and indicates that the complex seawater environment does not interfere with the general trends observed in cyclodextrin-based extraction. Fluorophore ratios in this case (Table 2) confirm the high levels of efficiency observed for the methyl- $\beta$-cyclodextrin host in promoting fluorophore extraction into the aqueous seawater.

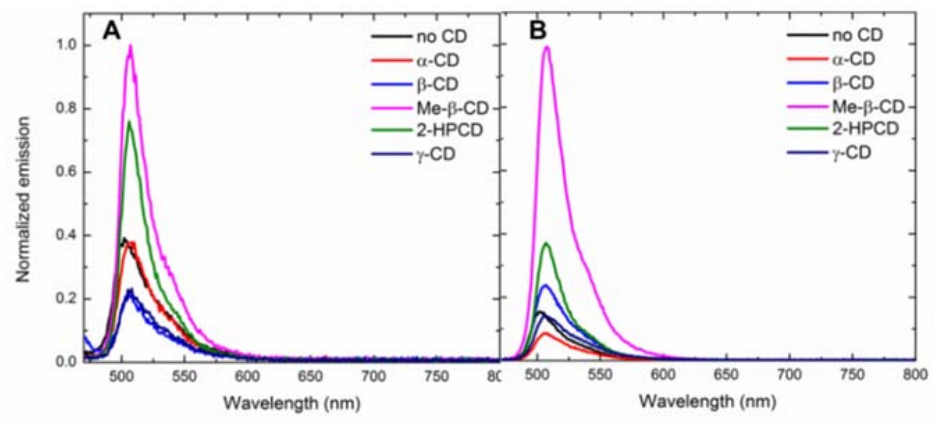

Fig 4 A comparison of the amount of fluorophore 4 extracted from the oil layer into the aqueous layer for (A) oil spill oil and (B) tar ball oil (460 $\mathrm{nm}$ excitation) 
Table 2: Fluorophore ratios ${ }^{a b}$

\begin{tabular}{|c|c|c|}
\hline Host & Oil spill oil & Tar ball oil \\
\hline$\alpha$-cyclodextrin & $0.92 \pm 0.01$ & $0.64 \pm 0.00$ \\
\hline$\beta$-cyclodextrin & $0.66 \pm 0.01$ & $1.53 \pm 0.01$ \\
\hline methyl- $\beta$-cyclodextrin & $1.80 \pm 0.02$ & $5.50 \pm 0.01$ \\
\hline 2-hydroxypropyl- $\beta$-cyclodextrin & $1.28 \pm 0.02$ & $1.97 \pm 0.01$ \\
\hline$\gamma$-cyclodextrin & $0.64 \pm 0.01$ & $1.05 \pm 0.02$ \\
\hline
\end{tabular}

a All fluorophore ratios were calculated according to Equation 2

$b$ The reported results represent an average of 4 trials.

After successful extraction of both the donor and fluorophore into the cyclodextrin-containing aqueous layers, energy transfer between the two small molecules can then occur. This energy transfer was most efficient for $\beta$-cyclodextrin and $\gamma$-cyclodextrin solutions that had been extracted from oil spill oil, with $56 \%$ and $51 \%$ energy transfer efficiencies observed (Table 3, Figure 5). Interestingly, such energy transfer was less efficient in the methyl- $\beta$-cyclodextrin solutions, despite the fact that methyl- $\beta$ cyclodextrin was most efficient at extracting the two energy transfer partners into the aqueous environment. This result means that energy transfer efficiencies do not depend strictly on the concentration of the energy transfer partners (because the Me- $\beta$-cyclodextrin solution contains the highest amount of both donor and acceptor), a result that is in line with ample literature precedent [32-35]. There is also a strong possibility that the binding of one analyte affects the binding of a second analyte, either by promoting or disrupting that binding. This interaction between analytes may be one reason why the results obtained using methyl- $\beta$-cyclodextrin (which is known to bind both the donor and acceptor strongly) are lower than those obtained with $\beta$-cyclodextrin and $\gamma$-cyclodextrin.

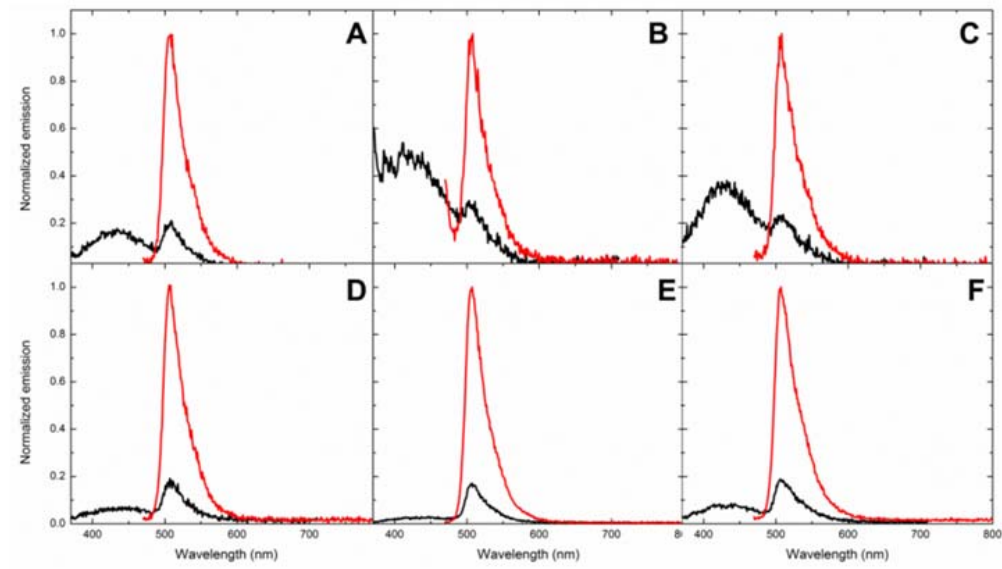

Fig 5 Energy transfer efficiencies measured in aqueous extracts from oil spill oil (A-C) and tar ball oil (DF) using an (A,D) $\alpha$-cyclodextrin host; $(\mathrm{B}, \mathrm{E}) \beta$-cyclodextrin host; and $(\mathrm{C}, \mathrm{F}) \gamma$-cyclodextrin host. The black line represents analyte excitation and the red line represents direct fluorophore excitation

Table 3: Aqueous energy transfer efficiencies in undoped oil-seawater samples ${ }^{a b}$

\begin{tabular}{|c|c|c|}
\hline Host & Oil spill oil & Tar ball oil \\
\hline
\end{tabular}




\begin{tabular}{|c|c|c|}
\hline$\alpha$-cyclodextrin & $38.6 \pm 0.2$ & $32.0 \pm 0.2$ \\
\hline$\beta$-cyclodextrin & $55.7 \pm 0.3$ & $23.6 \pm 0.1$ \\
\hline methyl- $\beta$-cyclodextrin & $30.8 \pm 0.4$ & $20.5 \pm 0.0$ \\
\hline 2-hydroxypropyl- $\beta$-cyclodextrin & $29.4 \pm 3.0$ & $25.3 \pm 0.1$ \\
\hline$\gamma$-cyclodextrin & $51.2 \pm 1.4$ & $29.0 \pm 0.3$ \\
\hline
\end{tabular}

a All energy transfer efficiencies were calculated according to Equation 3

$b$ The reported results represent an average of 4 trials

Oil-water mixtures that were doped with small amounts of analytes 1-3 demonstrated qualitatively similar behaviors in analyte extraction and fluorophore extraction compared to the undoped mixtures, likely a result of the fact that the large quantities of innate toxicants found in these crude mixtures dominates the amount of added aromatic analyte. Summary tables of results for all doped solutions are found in the ESI. Figure 6 highlights results obtained for energy transfer investigations in analyte-doped aqueous extracts, and indicates that the energy transfer peak is more clearly defined in the cyclodextrin containing solutions as a result of the known propensity of cyclodextrin to promote the desired intermolecular interactions.

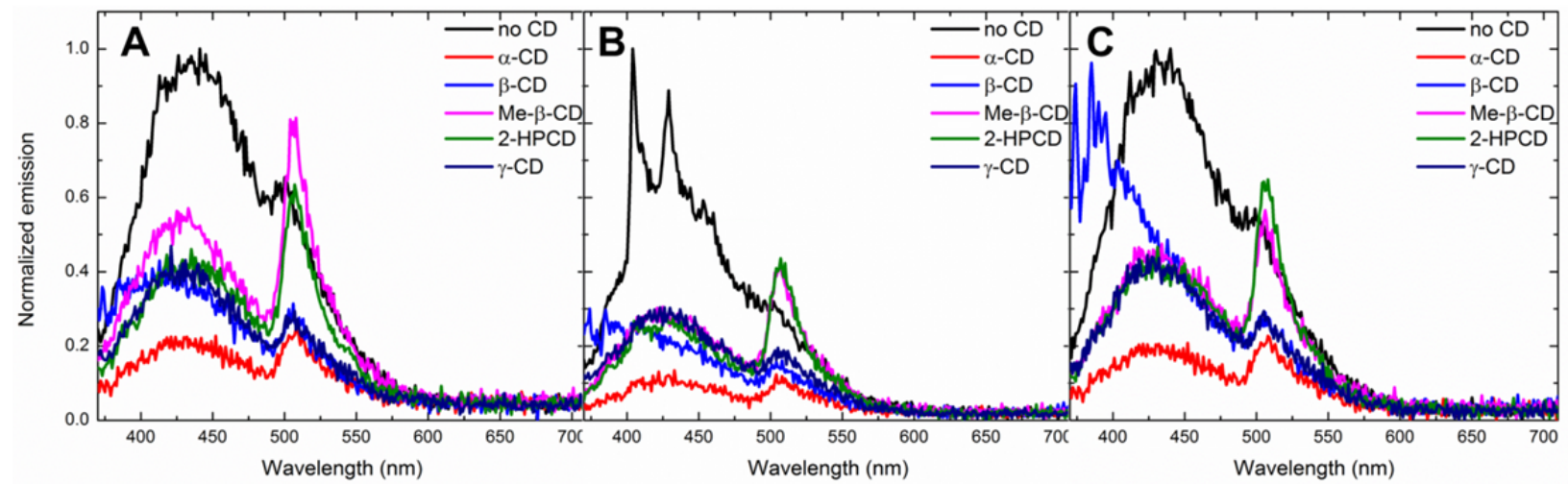

Fig 6 Aqueous energy transfer efficiencies measured in extracts from oil spill oil with (A) analyte 1; (B) analyte 2; and (C) analyte $\mathbf{3}$ doped into the oil-water mixtures

The beneficial effects of cyclodextrin in promoting the desired energy transfer were quantified according to Equation 4:

Energy enhancement $=F l_{\mathrm{CD}} / F l_{\text {blank }}$

where $F l_{\mathrm{CD}}$ is the fluorescence emission of the fluorophore from analyte excitation in the presence of a cyclodextrin host and $F l_{\text {blank }}$ is the fluorescence emission of the fluorophore from analyte excitation in the absence of the cyclodextrin host. Selected results are summarized in Table 4, and highlight that for oil spill oil samples, the energy transfer peak is more intense in the absence of cyclodextrin (as indicated by ratios less than 1), despite the fact that the peaks are substantially better defined (Figure 6). In tar ball oil extracts, in contrast, the cyclodextrins markedly improve the energy transfer efficiencies (Figure 7), with the exception of $\alpha$-cyclodextrin which led to a slight decrease in energy transfer efficiencies.

Table 4: Energy enhancement values for oil spill oil extracts

\begin{tabular}{|l|l|l|l|l|l|}
\hline analyte & $\alpha$-CD & $\beta$-CD & Me- $\beta$-CD & 2 -HPCD & $\gamma$-CD \\
\hline none & $0.53 \pm 0.02$ & $0.54 \pm 0.02$ & $0.82 \pm 0.05$ & $0.56 \pm 0.07$ & $0.49 \pm 0.03$ \\
\hline $\mathbf{1}$ & $0.61 \pm 0.00$ & $0.54 \pm 0.01$ & $0.88 \pm 0.00$ & $0.74 \pm 0.01$ & $0.55 \pm 0.01$ \\
\hline
\end{tabular}




\begin{tabular}{|l|l|l|l|l|l|}
\hline $\mathbf{2}$ & $0.43 \pm 0.03$ & $0.54 \pm 0.03$ & $0.76 \pm 0.08$ & $0.69 \pm 0.12$ & $0.57 \pm 0.06$ \\
\hline $\mathbf{3}$ & $0.48 \pm 0.01$ & $0.62 \pm 0.01$ & $0.79 \pm 0.02$ & $0.78 \pm 0.03$ & $0.61 \pm 0.03$ \\
\hline
\end{tabular}

$a$ All energy transfer efficiencies were calculated according to Equation 4

$b$ The reported results represent an average of 4 trials

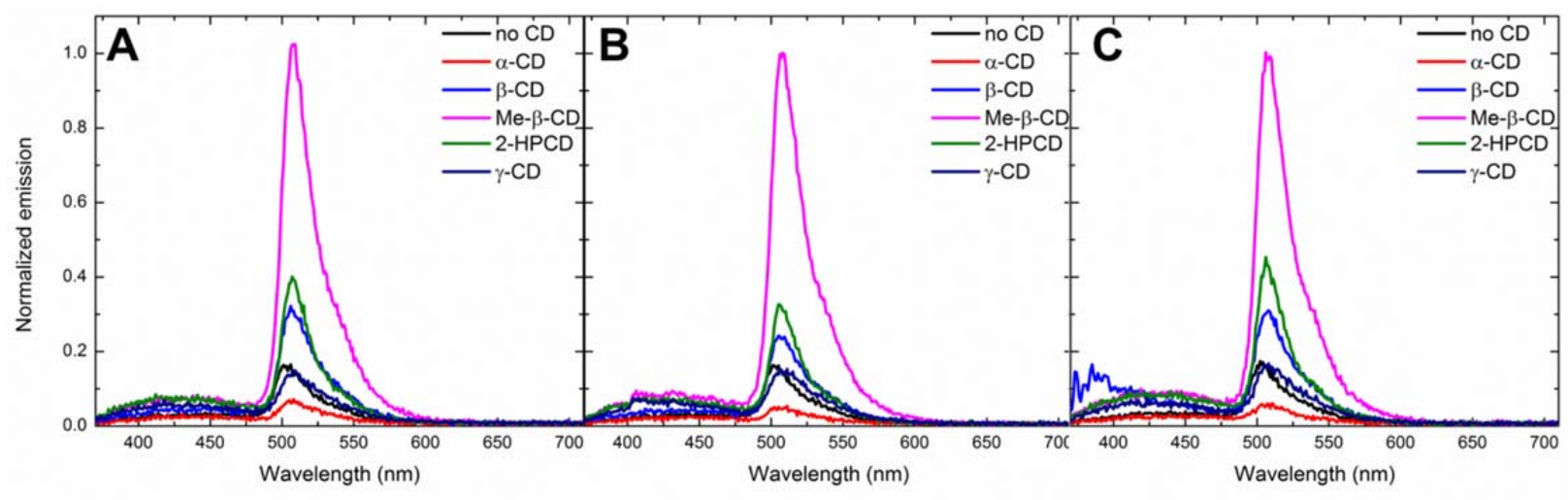

Fig 7 Aqueous energy transfer efficiencies measured in extracts from tar ball oil with (A) analyte 1; (B) analyte 2; and (C) analyte $\mathbf{3}$ doped into the oil-water mixtures

More interesting results were obtained by comparing the energy transfer results obtained in crude seawater to those obtained using phosphate buffered saline (PBS). PBS is generally used as a mimic of biological fluids, with a moderately high salt content and a $\mathrm{pH}$ of 7.4 [17]. Seawater, in contrast, has a notably higher salt content and a mildly elevated average $\mathrm{pH}$ of approximately 8.0 [18]. These differences translated into notable differences in the observed energy transfer efficiencies in aqueous extracts that had been doped with analyte 1, and these differences were quantified according to the following Equation:

Energy transfer ratio $=F l_{\mathrm{PBS}} / F l_{\text {seawater }}$

where $\mathrm{Fl}_{\mathrm{PBS}}$ is the integrated fluorophore emission from analyte excitation in a PBS solution and $\mathrm{Fl}_{\text {seawater }}$ is the integrated fluorophore emission from analyte excitation in crude seawater. Selected results of these experiments are summarized in Table 5. In the absence of any cyclodextrin host, energy transfer was less efficient in PBS compared to the energy transfer in seawater (indicated by energy transfer ratios less than 1), which highlights the significant potential of this energy transfer in developing practical detection systems that operate in complex environments such as contaminated seawater. Cyclodextrin addition partially reversed that preference for the oil spill oil extracts, but generally maintained the preference for aqueous solution extracted from tar ball oil. Reasons for these observed differences are currently under investigation in our laboratory, but preliminary results point to the importance of high salt content in promoting the favorable interactions required for efficient analyte-to-fluorophore energy transfer.

Table 5: Ratios of energy transfer efficiencies in PBS to energy transfer efficiencies in seawater ${ }^{a b}$

\begin{tabular}{|c|c|c|}
\hline Host & Oil spill oil & Tar ball oil \\
\hline no cyclodextrin & $0.39 \pm 0.01$ & $0.69 \pm 0.00$ \\
\hline$\alpha$-cyclodextrin & $1.06 \pm 0.01$ & $1.28 \pm 0.02$ \\
\hline$\beta$-cyclodextrin & $0.94 \pm 0.01$ & $0.28 \pm 0.01$ \\
\hline methyl- $\beta$-cyclodextrin & $1.15 \pm 0.02$ & $0.38 \pm 0.00$ \\
\hline
\end{tabular}




\begin{tabular}{|c|c|c|}
\hline 2-hydroxypropyl- $\beta$-cyclodextrin & $0.86 \pm 0.01$ & $0.82 \pm 0.00$ \\
\hline$\gamma$-cyclodextrin & $1.45 \pm 0.02$ & $1.01 \pm 0.01$ \\
\hline
\end{tabular}

a All ratios were calculated according to Equation 5 in oil-water mixtures that had been doped with analyte $\mathbf{1}([\mathbf{1}]]=22 \mu \mathrm{M})$. All solutions were excited at $360 \mathrm{~nm}$.

$b$ The reported results represent an average of 4 trials

Interestingly, replacing the seawater with phosphate buffer (rather than phosphate buffered saline) led to analyte comparison ratios that were approximately equivalent to what was observed in the oil-seawater systems (see ESI for more details). Fluorophore comparison ratios, in contrast, were markedly decreased in the phosphate buffer compared to what was observed in the seawater system (Table 6). Interactions between the fluorophore and the cyclodextrin host are likely to be dominated by hydrogen bonding interactions (as a result of the plethora of hydrogen bond acceptor groups found on the fluorophore structure), whereas the interactions between the analytes and cyclodextrins are likely to be dominated more by hydrophobic association and binding in the hydrophobic cyclodextrin cavity. The buffer system in this case likely disrupts the favorable hydrogen bonding between the fluorophore and cyclodextrin hosts, leading to the observed decrease in fluorophore extraction efficiencies.

Table 6: Fluorophore extraction efficiencies observed in complex oil-water systems. ${ }^{\text {a }}$

\begin{tabular}{|l|l|l|l|l|}
\hline Matrix & \multicolumn{2}{|c|}{ 2-HPCD } & \multicolumn{2}{c|}{$\gamma$-CD } \\
\hline & seawater & buffer & seawater & buffer \\
\hline oil spill oil & $85.2 \pm 0.3$ & $57.7 \pm 0.3$ & $74.9 \pm 0.2$ & $60.6 \pm 0.1$ \\
\hline tar ball oil & $40.4 \pm 0.1$ & $9.9 \pm 0.1$ & $25.0 \pm 0.4$ & $8.6 \pm 0.1$ \\
\hline
\end{tabular}

a All fluorophore extraction efficiencies were calculated as the ratio of $F l_{\mathrm{aq}} / F l_{\text {total }} \times 100 \%$, where $F l_{\mathrm{aq}}$ is the integrated emission of the fluorophore in the aqueous layer and $F l_{\text {total }}$ is the total emission of the fluorophore in both layers. The results represent an average of 4 trials.

A more extensive solvent screen was conducted on undoped samples from oil spill oil using $10 \mathrm{mM} \gamma-$ cyclodextrin, and included the following solvent systems: an ethanol/water mixture $(1: 1 \mathrm{vol} / \mathrm{vol}), 1 \mathrm{M}$ guanidinium hydrochloride in ultrapure water, $1 \mathrm{M}$ sodium chloride in ultrapure water, phosphate buffer, phosphate-buffered saline, and ultrapure water. These results (Table 7) highlight a strong solvent dependence for the extraction of photophysically active analytes from undoped oil, with sodium chloride acting to promote the extraction of the analytes (compare phosphate-buffered saline vs. phosphate buffer or $1 \mathrm{M} \mathrm{NaCl}$ vs $1 \mathrm{M}$ guanidinium hydrochloride), likely as a result of the known ability of $\mathrm{NaCl}$ to promote hydrophobic association [36]. These results can be seen more clearly in Figure 8, which compares the amount of photophysically active compound found in different aqueous layers, and highlights the beneficial role of sodium chloride in promoting the desired extraction. These results also show slight improvements using an ethanol co-solvent compared to ultrapure water (Figure 8C), in line with literature reports that show that the presence of ethanol promotes binding of analytes in the cyclodextrin cavity [37]. Moreover, the only solvent systems that led to a quantifiable energy transfer peak were those that contained sodium chloride (Table 7), which is further confirmed by the ability of these solvent systems to promote stronger fluorescence via direct excitation of the fluorophore (Figure 9).

Table 7: Solvent screen for PAH extraction and aqueous detection from oil spill oil with $\gamma$-CD ${ }^{\mathrm{a}}$

\begin{tabular}{|c|c|c|}
\hline Solvent & Analyte comparison & Aqueous layer energy transfer \\
\hline $1: 1$ ethanol/water mix & $71.8 \pm 0.1$ & $d$ \\
\hline 1 M guanidinium hydrochloride & $62.4 \pm 0.2$ & $d$ \\
\hline
\end{tabular}




\begin{tabular}{|c|c|c|}
\hline $1 \mathrm{M} \mathrm{NaCl}^{\mathrm{b}}$ & $68.4 \pm 0.1$ & $88.2 \pm 2.3$ \\
\hline Phosphate buffer & $62.5 \pm 0.2$ & $d$ \\
\hline Phosphate buffered saline & $74.5 \pm 0.2$ & $95.6 \pm 3.2$ \\
\hline Ultrapure water $^{\mathrm{c}}$ & $67.7 \pm 0.4$ & $78.0 \pm 0.7$ \\
\hline
\end{tabular}

a All analyte comparison ratios were calculated using Equation 1, and energy transfer efficiencies were calculated using Equation 3, and the results represent an average of 4 trials.

b Salt solutions were $1 \mathrm{M}$ in ultrapure water.

c Ultrapure water was obtained using a Milli-Q filtration system.

d No real energy transfer peak was observed

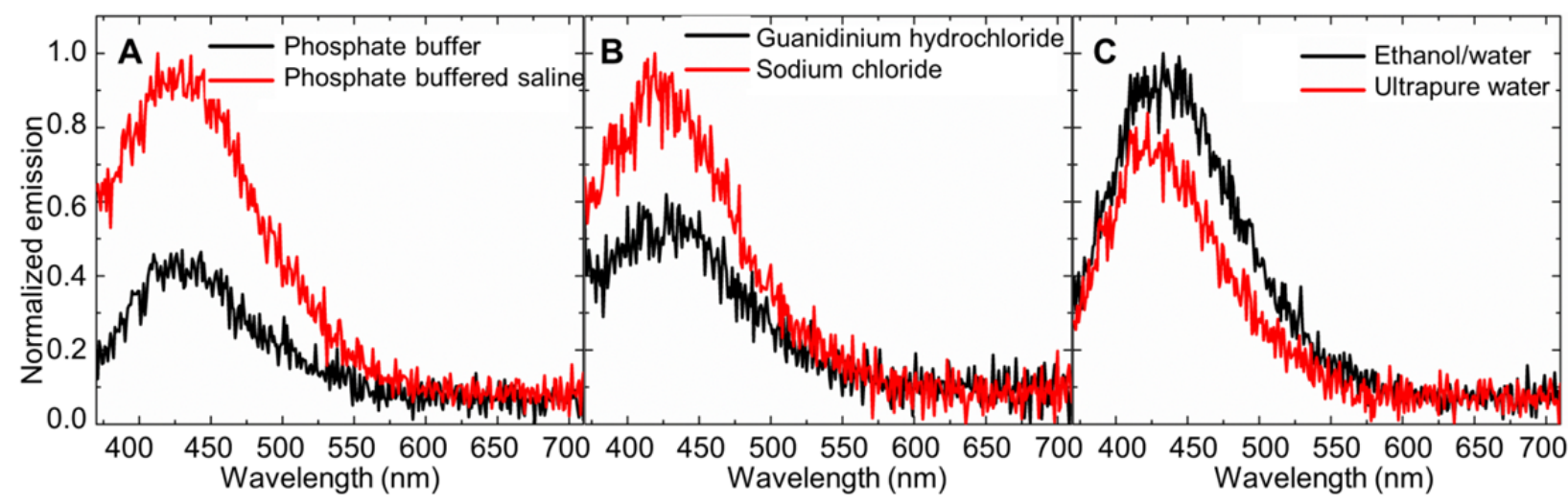

Fig 8 Solvent comparisons for the extraction of photophysically active components from undoped oil spill oil with $\gamma$-cyclodextrin solutions

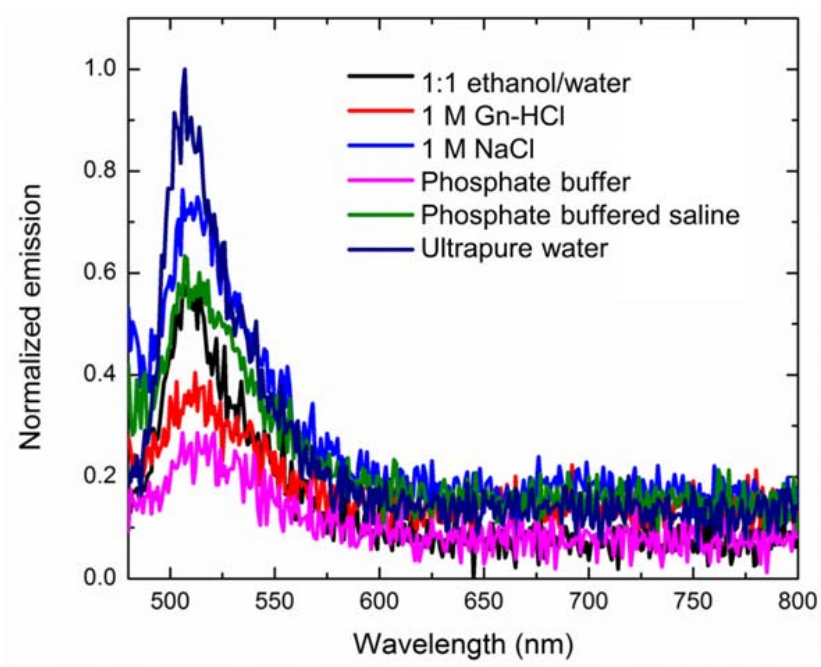

Fig 9 Aqueous fluorescence emission of fluorophore 4 with $10 \mathrm{mM} \gamma$-cyclodextrin in varying solvent compositions, extracted from undoped oil spill oil. $\lambda_{\max }$ excitation $=460 \mathrm{~nm}$.

In general, non-covalent cyclodextrin based complexes rely on a variety of intermolecular interactions, including hydrophobic association, intermolecular hydrogen bonding, and electrostatic and steric complementarity [38]. Many of these forces are known to depend strongly on experimental conditions, including in particular the composition of the solvent and the presence of solvent additives [39-41]. In this system, cyclodextrin complex formation is required for three distinct roles: extraction of aromatic 
toxicants from the oil into the cyclodextrin-containing aqueous layer, extraction of a high quantum yield fluorophore from the oil into the cyclodextrin-containing aqueous layer, and facilitation of non-covalent energy transfer from the aromatic analyte donor to the fluorophore acceptor. We have seen that each of these roles is affected by the specific solvent composition, with hydrophobic association predominating as a key intermolecular force in these systems $[42,43]$.

\section{CONCLUSION}

We have demonstrated that cyclodextrins can be very useful in environmental remediation efforts in that they can be used for tandem PAH extraction and detection. Solvent effects were investigated and showed that the complex intermolecular interactions responsible for analyte extraction, fluorophore extraction, and non-covalent energy transfer are strongly solvent dependent in ways that are often difficult to predict. Additionally, the fact that efficient extraction and detection occurred in tar ball and oil spill samples collected from spill sites and seawater from Narragansett Bay in Rhode Island emphasizes the strength and general applicability of these non-covalent interactions. Taken together, these results highlight the potential of cyclodextrin-based systems in real-world environmental remediation efforts.

\section{ACKNOWLEDGEMENTS}

Funding for this research was provided by the Gulf of Mexico Research Initiative

[1] McNutt, M. K., Chu, S., Lubchenco, J., Hunter, T., Dreyfus, G., Murawski, S. A., Kennedy, D. M.: Applications of science and engineering to quantify and control the Deepwater Horizon oil spill. Proc. Natl. Acad. Sci. U.S.A. 109, 20222-20228 (2012).

[2] Ruddy, B. M., Huettel, M., Kostka, J. E., Lobodin, V. V., Bythell, B. J., McKenna, A. M., Aeppli, C., Reddy, C. M., Nelson, R. K., Marshall, A. G., Rodgers, R.P.: Targeted petroleomics: analytical investigation of Macondo well oil oxidation products from Pensacola beach. Energy \& Fuels 28, 4043-4050 (2014).

[3] Hall, G. J., Frysinger, G. S., Aeppli, C., Carmichael, C. A., Gros, J., Lemkau, K. L., Nelson, R. K., Reddy, C. M.: Oxygenated weathering products of Deepwater Horizon oil come from surprising precursors. Marine Pollution Bull. 75, 140-149 (2013).

[4] Krahn, M. M., Ylitalo, G. M., Buzitis, J., Bolton, J. L., Wigren, C. A., Chan, S.-L., Varanasi, U.: Analyses for petroleum-related contaminants in marine fish and sediments following the Gulf oil spill. Marine Pollution Bull. 27, 285-292 (1993).

[5] John, G. F., Yin, F., Mulabagal, V., Hayworth, J. S., Clement, T. P.: Development and application of an analytical method using gas chromatography/triple quadrupole mass spectrometry for characterizing alkylated chrysenes in crude oil samples. Rapid Commun. Mass Spectrometry 28, 948-956 (2014).

[6] Aeppli, C., Carmichael, C. A., Nelson, R. K., Lemkau, K. L., Graham, W. M., Redmond, M. C., Valentine, D. L., Reddy, C. M.: Oil weathering after the Deepwater Horizon disaster led to the formation of oxygenated residues. Environ. Sci. Technol. 46, 8799-8807 (2012).

[7] Parker, A. M., Ferrer, I., Thurman, E. M., Rosario-Ortiz, F. L., Linden, K. G.: Determination of COREXIT components used in the Deepwater Horizon cleanup by liquid chromatography-ion trap mass spectrometry. Anal. Methods 6, 5498-5502 (2014).

[8] Serio, N., Chanthalyma, C., Prignano, L., Levine, M.: Cyclodextrin-enhanced extraction and energy transfer of carcinogens in complex oil environments. ACS Appl. Mater. Interfaces 5, 11951-11957 (2013).

[9] Serio, N., Levine, M.: Efficient extraction and detection of aromatic toxicants from crude oil and tar balls using multiple cyclodextrin derivatives. Marine Pollution Bull. 95, 242-247 (2015).

[10] Hamai, S.: Complex formation of tetrakis(4-sulfonatophenyl)porphyrin with $\gamma$-cyclodextrin, phenylalanine, and tryptophan in aqueous solution. J. Inclusion Phenom. Macrocyclic Chem. 67: 471-481 (2010).

[11] Hamai, S.: Ternary inclusion complexes of $\gamma$-cyclodextrin with resorufin and organic cations in aqueous solution. Bull. Chem. Soc. Japan 80, 1527-1533 (2007). 
[12] Serio, N., Prignano, L., Peters, S., Levine, M.: Detection of medium-sized polycyclic aromatic hydrocarbons via fluorescence energy transfer. Polycyclic Aromatic Compounds 34, $561-572$ (2014).

[13] Serio, N., Chanthalyma, C., Prignano, L., Levine, M.: Cyclodextrin-promoted energy transfer for broadly applicable small-molecule detection. Supramol. Chem. 26, 714-721 (2014).

[14] Serio, N., Miller, K., Levine, M.: Efficient detection of polycyclic aromatic hydrocarbons and polychlorinated biphenyls via three-component energy transfer. Chem. Commun. 49, 4821-4823 (2013).

[15] Mako, T., Marks, P., Cook, N., Levine, M.: Fluorescent detection of polycyclic aromatic hydrocarbons in ternary cyclodextrin complexes. Supramol. Chem. 24, 743-747 (2012).

[16] Serio, N., Chanthalyma, C., Peters, S., Levine, D., Levine, M.: 2-Hydroxypropyl beta-cyclodextrin for the enhanced performance of dual function extraction and detection systems in complex oil environments. J. Inclusion Phenomena Macrocyclic Chem. 81, 341-346 (2015).

[17] http://www.medicago.se/sites/default/files/pdf/productsheets/PBS_Buffer_v._01.pdf

[18] Robert-Baldo, G. L., Morris, M. J., Byrne, R. H.: Spectrophotometric determination of seawater pH using phenol red. Anal. Chem. 57, 2564-2567 (1985).

[19] Pytkowicz, R. M., Atlas, E., Culberson, C. H.: Chemical equilibrium in sea water. ACS Symposium Series 18, 1-24 (1975).

[20] Breslow, R.: Hydrophobic effects on simple organic reactions in water. Acc. Chem. Res. 24, 159-164 (1991).

[21] Breslow, R.: The hydrophobic effect in reaction mechanism studies and in catalysis by artificial enzymes. J. Phys. Org. Chem. 19, 813-822 (2006).

[22] Riley, K. E., Hobza, P.: On the importance and origin of aromatic interactions in chemistry and biodisciplines. Acc. Chem. Res. 46, 927-936 (2013).

[23] Schneider, H.-J., Yatsimirsky, A. K.: Selectivity in supramolecular host-guest complexes. Chem. Soc. Rev. 37, 263-277 (2008).

[24] Li, S., Xu, Y., Shen, Q., Liu, X., Lu, J., Chen, Y., Lu, T., Luo, C., Luo, X., Zheng, M., Jiang, H.: Non-covalent interactions with aromatic rings: current understanding and implications for rational drug design. Curr. Pharmaceutical Design 19, 6522-6533 (2013).

[25] Shepherd, J. L., Kell, A., Chung, E., Sinclar, C. W., Workentin, M. S., Bizzotto, D.: Selective reductive desorption of a SAM-coated gold electrode revealed using fluorescence microscopy. J. Am. Chem. Soc. 126, 83298355 (2004).

[26] Yin, F., Hayworth, J. S., Clement, T. P. A tale of two recent spills-comparison of 2014 Galveston Bay and 2010 deepwater horizon oil spill residues. PLoS One 10, e0118098/1-e0118098/17 (2015).

[27] Mendoza, W. G., Riemer, D. D., Zika, R. G. Application of fluorescence and PARAFAC to assess vertical distribution of subsurface hydrocarbons and dispersant during the Deepwater Horizon oil spill. Environ. Sci. Process Impacts 15, 1017-1030 (2013).

[28] Aeppli, C., Reddy, C. M., Nelson, R. K., Kellermann, M. Y., Valentine, D. L. Recurrent oil sheens at the Deepwater Horizon disaster site fingerprinted with synthetic hydrocarbon drilling fluids. Environ. Sci. Technol. 47, 8211-8219 (2013).

[29] McKenna, A. M., Nelson, R. K., Reddy, C. M., Savory, J. J., Kaiser, N. K., Fitzsimmons, J. E., Marshall, A. G., Rodgers, R. P. Expansion of the analytical window for oil spill characterization by ultrahigh resolution mass spectrometry: beyond gas chromatography. Environ. Sci. Technol. 47, 7530-7539 (2013).

[30] Wise, J. P., Jr., Wise, J. T. F., Wise, C. F., Wise, S. S., Gianios, C., Jr., Xie, H., Thompson, W. D., Perkins, C., Falank, C., Wise, J. P., Sr. Concentrations of the genotoxic metals, chromium and nickel, in whales, tar balls, oil slicks, and released oil from the Gulf of Mexico in the immediate aftermath of the Deepwater Horizon oil crisis: Is genotoxic metal exposure part of the Deepwater Horizon legacy? Environ. Sci. Technol. 48, 2997-3006 (2014). 
[31] Warnock, A. M., Hagen, S. C., Passeri, D. L. Marine tar residues: a review. Water, Air, \& Soil Pollution 226, 124 (2015).

[32] Chen, Z., Chang, K., Capraro, B. R., Zhu, C., Hsu, C.-J., Baumgart, T.: Intradimer/intermolecular interactions suggest autoinhibition mechanism in Endophilin A1. J. Am. Chem. Soc. 136, 4557-4564 (2014).

[33] Schlosser, M., Lochbrunner, S.: Exciton migration by ultrafast Foerster transfer in highly doped matrixes. J. Phys. Chem. B 110, 6001-6009 (2006).

[34] Tanabe, S., Suzuki, K., Soga, N., Hanada, T.: Mechanisms and concentration dependence of Tm3+ blue and Er3+ green up-conversion in codoped glasses by red-laser pumping. J. Luminescence 65, 247-255 (1995).

[35] Fisher, D. L., Harper, J., Sailor, M. J.: Energy transfer quenching of porous Si photoluminescence by aromatic molecules. J. Am. Chem. Soc. 117, 7846-7847 (1995).

[36] Arakawa, T., Kita, Y., Ejima, D., Gagnon, P. Solvent modulation of column chromatography. Protein \& Peptide Letters 15, 544-555 (2008).

[37] Patonay, G., Fowler, K., Shapira, A., Nelson, G., Warner, I. M. Cyclodextrin complexes of polyaromatic hydrocarbons in the presence of aliphatic alcohols. J. Inclusion Phenom. 5, 717-723 (1987).

[38] Schneider, H.-J. Binding mechanisms in supramolecular complexes. Angew. Chem. Int. Ed. 48, 3924-3977 (2009).

[39] Connors, K. The stability of cyclodextrin complexes in solution. Chem. Rev. 97, 1325-1357 (1997).

[40] Loftsson, T., Brewster, M. E. Pharmaceutical applications of cyclodextrins. 1. Drug solubilization and stabilization. J. Pharm. Sci. 85, 1017-1025 (1996).

[41] Müller, B. W., Albers, E. Effect of hydrotropic substances on the complexation of sparingly soluble drugs with cyclodextrin derivatives and the influence of cyclodextrin complexation on the pharmacokinetics of the drugs. J. Pharm. Sci. 80, 599-604 (1991).

[42] Biedermann, F., Nau, W. M., Schneider, H.-J. The hydrophobic effect revisited - studies with supramolecular complexes imply high-energy water as a noncovalent driving force. Angew. Chem. Int. Ed. 53, 11158-11171 (2014).

[43] Ross, P. D., Rekharsky, M. V. Thermodynamics of hydrogen bond and hydrophobic interactions in cyclodextrin complexes. Biophys. J. 71, 2144-2154 (1996). 